\author{
Agustín Darias Marrero \\ Universidad de Las Palmas de Gran Canaria \\ agustin.darias@ulpgc.es
}

\title{
EL ESTRÉS EN SITUACIONES DE INTERPRETACIÓN: UN ESTUDIO COMPARATIVO ENTRE INTERPRETACIÓN DE CONFERENCIAS E INTERPRETACIÓN PARA LOS SERVICIOS PÚBLICOS
}

DOl: http://dx.doi.org/10.12775/RP.2020.006

\begin{abstract}
Resumen: Este trabajo presenta un estudio de casos de interpretación de conferencias y de interpretación para los Servicios Públicos, en el que se analiza el corpus objeto de estudio compuesto por distintas situaciones de interpretación, mediante entrevistas semiestructuradas a intérpretes, guiándoles en la cumplimentación de la herramienta metodológica empleada: una ficha de análisis de los factores constituyentes de la situación mediada por intérpretes. El objetivo último reside en identificar qué factores son susceptibles de producir estrés y qué competencias se requieren al intérprete en los distintos contextos de interpretación. En conclusión, se constata que los factores de estrés varían de un tipo de interpretación a otro, al igual que algunas de las competencias requeridas.
\end{abstract}

Palabras clave: situaciones de interpretación, estrés, interpretación de conferencias, ruedas de prensa, interpretación para Servicios Públicos, interpretación médica

\section{Introducción}

Con el propósito de abordar el tema del estrés en la interpretación, conceUbido psicológicamente como la percepción de un sujeto de que las exigencias del entorno exceden sus propios recursos disponibles para hacerles 
frente (Lazarus 2000, Riccardi, Marinuzzi, Zecchin 1998, Kurz 2003), en este trabajo se ha realizado una revisión teórica de algunos estudios relativos al estrés en interpretación, tanto en Interpretación de conferencias (IC) (AIIC 2002, Jiménez Ivars, Pinazo Calatayud 2002, Kurz 2003, Moser-Mercer, Künzli, Korac 1998, Renau Michavila 2010, Riccardi, Marinuzzi, Zecchin 1998) como en Interpretación para los Servicios Públicos (ISSPP) (Corsellis 2010, Valero Garcés 2006, 2008). Tras considerar las aportaciones teóricas de los autores consultados, que se tratarán en los apartados dedicados a los correspondientes tipos de interpretación (Jiménez Ivars 2002), queda claro que el estrés forma parte inherente de la interpretación. Del mismo modo, parece que se podría afirmar la hipótesis de partida de este trabajo, según la cual la ISSPP comporta aspectos que generarían potencialmente más estrés o, sobre todo, diferente al de la IC.

Así, con la intención de comprobar la veracidad de esta afirmación, desde el punto de vista metodológico, se han contemplado concepciones etnográficas (Bendazzoli 2010) apoyándonos en investigaciones teóricas previas (Abril Martí 2004, Alexieva 1997, Darias Marrero 2006, 2009, Gile 1989, Jiménez Ivars 2002, 2012, Pöchhacker 2003, 2004) centradas en los contextos comunicativos en los que evolucionan los intérpretes, es decir, que estudian las situaciones extralingüísticas mediadas por intérpretes, así como sus taxonomías y factores constituyentes.

Por tanto, nuestra metodología, tal y como se explicará en detalle más adelante, se ha basado en aplicar a un corpus de situaciones de interpretación una herramienta de análisis consistente en tres fichas. Estas incluyen los factores situacionales, interpersonales e informacionales básicos que constituyen todo evento comunicativo mediado por intérpretes. Con el fin de intentar identificar cuáles de esos factores son susceptibles de generar estrés se han realizado entrevistas semiestructuradas (Böser 2016) a intérpretes que trabajan en los distintos ámbitos de interpretación tomados como referentes (IC e ISSPP).

Por último, a la vista de los resultados del estudio llevado a cabo, se extraerán algunas conclusiones para, en la medida de lo posible, matizar la afirmación inicial, así como contribuir a la definición de distintos perfiles profesionales de intérprete, tras haber observado que se requieren diversas tareas a los intérpretes en uno u otro tipo de interpretación. 


\section{Estrés en IC}

A continuación, nos centraremos en el estrés, que se trata separadamente tan solo a efectos de la exposición, ya que somos conscientes de que toda consideración aislada de una de las múltiples dimensiones que incide en el proceso de la interpretación resultaría artificial, puesto que el proceso cognitivo que se realiza durante una interpretación es altamente complejo tan solo por el simple hecho de movilizar al mismo tiempo todas las competencias necesarias para su ejecución.

En efecto, una de las características más importantes de la interpretación como proceso o actividad mental es la rapidez y simultaneidad de las operaciones que se llevan a cabo (Collados Aís, Fernández Sánchez, Stévaux 2001: 46).

Todas las tareas cognitivas e intelectuales vinculadas con la recepción y la comprensión del discurso original, con la producción del discurso traducido y con la supervisión de la calidad de la expresión producida coinciden en el tiempo, con lo que la complejidad de simultanear tareas cognitivas altamente complejas produce estrés.

La interpretación resulta, así, una prestación compleja (Moser-Mercer, Künzli, Korac 1998: 47) que se realiza, además, en unas condiciones que se podrían calificar de extremas. En efecto, parece obvio que, en las condiciones del ejercicio profesional de la interpretación, el intérprete está inmerso en el contexto situacional amplio del acto comunicativo, en presencia de los interlocutores, y debe procesar una abundante oferta informativa en tiempo real y simultaneando múltiples actividades cognitivas complejas. No en vano la profesión de intérprete ha sido calificada como estresante por muchos autores, según Renau Michavila (2010). Asimismo, en la literatura sobre interpretación abundan las citas que afirman que el estrés parece ser intrínseco a la interpretación. Kurz (2003: 51) afirma que: "Several empirical studies have confirmed that simultaneous interpreting is indeed a high-stress occupation". Así, se suele dar por sentado que la interpretación de conferencias es una profesión con un alto nivel de exigencia.

Si bien, [...], son escasos los estudios empíricos sobre la repercusión del estrés en la interpretación, parece que existe consenso en considerar el estrés como un factor intrínseco a esta actividad, y que el éxito de la interpretación depende en gran medida de la capacidad de hacerle frente (Jiménez Ivars, Pinazo Calatayud 2002: 80). 
Entre los estudios empíricos que abordan la incidencia del estrés en la interpretación, el encargado en 2002 por la Asociación Internacional de Intérpretes de Conferencia (AIIC) se centra en la interpretación simultánea (IS) y trata parámetros psicológicos, fisiológicos, físicos y referentes al rendimiento, así como la interacción entre ellos. Entre los factores de estrés citados por los intérpretes destacan, entre otros, los oradores que leen textos, la velocidad del discurso, los acentos complicados, las condiciones de la cabina. Uno de los resultados apunta a una correlación débil entre las mediciones del estrés y el rendimiento de los intérpretes, de forma que los intérpretes altamente cualificados y motivados mantienen elevados niveles de prestación incluso en presencia de varios factores de estrés (AIIC 2002).

A pesar de ello, parece quedar claro que el ejercicio de la interpretación conlleva unos costes fisiológicos y psicológicos asociados (Moser-Mercer, Künzli, Korac 1998), y que las condiciones de ejercicio de la actividad, por ende, los factores que constituyen la situación comunicativa, son susceptibles de aumentar el estrés inherente a la IS, aunque los intérpretes pueden sobrellevarlo si cuentan con la experiencia suficiente:

While conference interpreters have learned to overcome their 'stage fright' with experience and have developed more tolerance for the stress involved in SI, student interpreters still grapple with numerous problems (Kurz 2003: 64).

De hecho, desde el punto de vista psicológico el estrés se entiende como "una relación particular entre la persona y su entorno valorado por la persona como una situación que le sobrepasa y pone en peligro su bienestar" (Lazarus 2000, Achotegui 2009), es decir, se produce un desequilibrio entre cómo percibe el sujeto las exigencias del contexto y su capacidad de respuesta. En el campo de la interpretación y en la misma línea, Kurz (2003: 59) considera que "stress depends on the complex relation between the individual and the environment", es decir, cuando el profesional percibe que las condiciones de la interpretación pueden sobrepasar su capacidad de solventar el reto que se le plantee, es susceptible de padecer respuestas fisiológicas, psicológicas y de comportamiento negativas. En cualquier caso, para que se desencadene la reacción de estrés es necesario que se perciba subjetivamente la situación como algo amenazante o peligroso, "the psychological processes caused by a perceived threat or danger" (AIIC 2002), ante el que no se dispone de los recursos suficientes para hacerle frente.

El estudio considerado aquí se centra en la IS. Con respecto al estrés en la interpretación consecutiva tanto dialógica como monológica (Jiménez 
Ivars 2002: 2-3), los factores inductores de estrés deben de estar relacionados en mayor medida con aspectos propios de esta modalidad de interpretación. En interpretación consecutiva, el mensaje se recibe en silencio, ante un público expectante por conocer el contenido del mensaje, y el intérprete escucha el texto una única vez, a medida que se desarrolla, con lo cual el sentido del mismo se va contextualizando paulatinamente. El intérprete debe disponer de un sistema de toma de notas eficaz para ayudar a su memoria auditiva con su memoria visual, "the visual reinforcement of notes per se" (Ilg, Lambert 1996: 84), y poder producir, inmediatamente después de que termine el discurso original, su exposición en el otro idioma.

Por tanto, factores situacionales, de índole externa, como la exposición directa del intérprete ante el público, la posible grabación audiovisual en comparecencias ante la prensa o simplemente las condiciones materiales que faciliten o no la prestación del intérprete (por ejemplo, poder ver al orador, tomar notas sentado o de pie, hablar con o sin micrófono...), además de otros factores de estrés compartidos con otras modalidades de interpretación, como personales o relativos a conocimientos temáticos y lingüísticos, pueden desencadenar reacciones de estrés en el intérprete. En efecto, ante una amenaza unas personas reaccionan mejor que otras, en función de su perfil psicológico y destaca, pues, la percepción subjetiva ante los estímulos, tanto internos como externos, que provocan el estrés (Riccardi, Marinuzzi, Zecchin 1998: 96). En resumen, el estrés es un factor constituyente de la prestación en IC, tanto simultánea como consecutiva, que se aprende a gestionar con la experiencia.

\section{Estrés en ISSPP}

Centrándonos a continuación en la ISSPP, cabe pensar que en ISSPP debe ocurrir lo mismo y en esa dirección apuntan los estudios mencionados por Valero Garcés (2006). Sin embargo, a diferencia de la IC, cuando se habla de estrés en ISSPP, se matiza y se le califica generalmente de estrés emocional, como veremos a continuación, por una serie de razones.

Algunas de las características de la ISSPP identificadas por Roberts (1994), además de por Abril Martí $(2004,2006)$, Corsellis (2010), El-Madkouri Maataoui (2008), Ticca, Traverso (2017), Valero Garcés, Dergam (2003), Valero Garcés $(2005,2006,2008,2014)$ y Wadensjö $(1992,1998)$, contribuyen a su clara distinción de la IC. Entre otras, destacamos la relevancia de conocer las necesidades de los usuarios extranjeros para facilitarles el acceso a los recursos provistos por los Servicios Públicos (Pöllabauer 2012), en 
situaciones que suelen ser de interpretación consecutiva dialógica (Jiménez Ivars 2002: 2) en ambos sentidos de la combinación lingüística del intérprete que, a su vez, es percibido a menudo como mediador menos invisible que el intérprete de conferencias al que se le asigna tradicionalmente un papel más neutro. "The interpreter's visibility has been a much-debated issue" (Setton, Dawrant 2016: 382), no obstante: "Given that neutrality is a notion concerning relations, the question concerning dialogue interpreters' activities must be: neutral in relation to whom and/or what?" (Wadensjö 1992: 268). Dadas las relaciones que se establecen en el marco general de la ISSPP, habría que mencionar, en primer lugar, el papel destacado de la mediación cultural y de la social entre los intervinientes en la situación concreta. Bobaila (2011: 194) alude a la confusión de competencias que sigue dándose en algunos países en los que el intérprete no sólo debe mediar lingüísticamente, sino también asegurar la mediación cultural, en base a las convenciones culturales de los intervinientes, y la mediación social, para entender y orientar las relaciones entre los Servicios Públicos y sus usuarios.

El intérprete mantiene un papel más visible que el intérprete de conferencias, debido a su participación activa en el proceso de comunicación, por ejemplo, gestionando los turnos de palabra. Este tipo de mediación pone en entredicho la posición de neutralidad que se suele requerir al intérprete en otras situaciones de interpretación, al tener que negociar continuamente y transmitir significados desde sus conocimientos y competencias interculturales (Darias Marrero, Vidal Luengo 2018: 197).

Así, parece obvio, en principio, que en los contextos profesionales de la ISSPP las diferencias interculturales son más palpables que en la IC en la que los oradores y participantes en conferencias o congresos con una componente internacional comparten de alguna manera una cultura común, la de las conferencias o congresos especializados internacionales (Gile 1989), conformando, así, una diacultura, como subgrupo cultural que comparte profesión e intereses (Vermeer 1983), en este caso, internacional:

Una cultura de grupo definida por la formación y los conocimientos profesionales compartidos, la experiencia común en un campo especializado y, por supuesto, la historia de interacciones entre los miembros [...]. Esta cultura de expertos en concreto rebasa las fronteras nacionales (Pöchhacker 2003: 119).

Por lo tanto, cuanto más uniforme sea la cultura de los interlocutores, menos escollos culturales tendrá que salvar el intérprete y, en consecuencia, 
Si en un encuentro más "universal", como un congreso internacional, el intérprete puede muy bien limitarse a actuar como mediador lingüístico "invisible", de un intérprete social se espera a menudo una mediación intercultural (Abril Martí 2004: 222).

Del mismo modo, cabe añadir a las diferencias interculturales aquellas relativas a la asimetría social (Jiménez Ivars 2002: 10), es decir, las diferencias de nivel social o de posición de poder entre los interlocutores. En efecto, uno de los participantes en el acto comunicativo interviene no en su nombre propio, sino como representante de un organismo público, lo que le inviste de un cierto grado de autoridad frente al otro que se encuentra en condiciones de desamparo por razones económicas, políticas o de salud. En ISSPP, los intérpretes median con frecuencia en situaciones críticas y que versan sobre temas delicados, íntimos o claramente dramáticos (guerras, migraciones, violencia, enfermedad), que inciden en un estado psicológico y emocional del usuario, cuando menos, frágil y en el que el intérprete no puede implicarse directamente, a pesar de que el usuario tiende a considerar al intérprete como su tabla de salvación, ya que es el único que parece entenderle. Los intérpretes, ante estas circunstancias, declaran sufrir trastornos psico-afectivos (Valero Garcés 2008: 164-168). También pueden sentir impotencia por no saber cómo actuar para ayudar e, incluso, miedo a herir a los usuarios de los Servicios Públicos.

Los mencionados desequilibrios socioculturales entre los locutores obligan a los intérpretes a gestionar un continuo trasvase entre sus respectivas convenciones socioculturales, lo que produce, en efecto, un estrés que parece ser de carácter más emocional que en la IC.

\section{Estudio comparativo de casos de IC e ISSPP}

Una vez constatada la presencia de estrés tanto en IC como en ISSPP, hemos procedido a comparar situaciones de IC e ISSPP, aun siendo conscientes de las divergencias entre ambos tipos de interpretación (Jiménez Ivars 2002: 5), con el fin de verificar la hipótesis inicial acerca de qué factores constituyentes de las situaciones de interpretación son susceptibles de inducir estrés en uno u otro tipo de interpretación. Se pretende también contribuir a delimitar las competencias de los perfiles profesionales en cuestión.

De este modo, se ha procedido metodológicamente en tres etapas. En la primera, se han caracterizado las situaciones de interpretación contempladas 
como objeto de estudio. En segundo lugar, se ha llevado a cabo una serie de entrevistas semiestructuradas a los intérpretes que mediaron en ellas, para que proporcionaran su feed-back retrospectivo sobre los factores de los eventos comunicativos que han supuesto mayor o menor fuente de estrés. Por último, se ha procedido a analizar y sintetizar las respuestas de los intérpretes con el fin de presentar resultados sucintos y legibles en tablas.

\subsection{Corpus de situaciones de interpretación}

Dada la gran dificultad de tipificar los contextos comunicativos que configuran el telón de fondo de la realidad de la interpretación puesto que "la situación comunicativa es única e irrepetible en cada acto de comunicación" (Lvóvskaya 1997: 54), en cuanto a temas, identidad de los participantes y modalidades de organización, nos hemos centrado en unas situaciones concretas extraídas de la realidad profesional, que constituyen el corpus contemplado en este trabajo.

Entonces, para definir las situaciones objeto de este estudio, como primera etapa metodológica, se revisaron teóricamente algunas de las clasificaciones existentes sobre encuentros mediados por intérpretes (Gile 1989, Alexieva 1997, Pöchhacker 2003, 2004, Abril Martí 2004, Jiménez Ivars 2002, 2012, Bendazzoli 2010). Tras esta somera revisión (Darias Marrero 2006: 126-170) que no comporta vocación alguna a la exhaustividad, se constata que el enfoque de estudiar desde el punto de vista extralingüístico las situaciones de interpretación resulta pertinente para los distintos autores consultados. Se trata de analizar y caracterizar los encuentros interlingües mediados, tomando como punto de partida el ejercicio profesional de la interpretación, con la intención de dar cuenta de una compleja realidad extralingüística en la que se entremezclan numerosos y variados factores.

En efecto, los criterios en los que están sustentadas las propuestas taxonómicas contempladas son heteróclitos. Por tanto, su empleo a efectos metodológicos para nuestros fines, se apoya en trabajos anteriores (Darias Marrero 2006: 255-272, 2009: 39-46), en los que se configuraron tres plantillas que recogen los factores esenciales que constituyen toda situación comunicativa mediada.

- Factores situacionales, que se refieren al marco espacio-temporal en sentido estricto y a los organizadores que son relevantes, sobre todo, en cuanto a la documentación y las condiciones materiales que ponen a disposición del intérprete. 
- Factores interpersonales, relativos a los actantes (Witte 2000: 163, 2005: 50) implicados en el acto comunicativo y sus objetivos comunicativos en el marco de sus relaciones socioculturales.

- Factores informacionales, que dan cuenta del contenido informativo del acto comunicativo y los intercambios orientados a su transmisión.

Así, las tres fichas de análisis respectivas permiten caracterizar situaciones de interpretación y dar cuenta de la mayor o menor incidencia de un factor en un caso concreto de encuentro mediado.

En este estudio, el corpus está constituido por situaciones de tipos de interpretación distintos, definidos fundamentalmente en función de la situación de uso y el objetivo de la comunicación, según Jiménez Ivars (2002: 5), pero en ambos casos, de IC y de ISSPP, son situaciones de interpretación consecutiva dialógica o bilateral (Jiménez Ivars 2002: 2). Para la IC, son ruedas de prensa en las que se ha hecho interpretación bilateral francés - español, por una parte, en tres ruedas de prensa con actores y actrices de cine de fama internacional en el marco de dos ediciones sucesivas de un festival internacional de cine y, por otra, dos ruedas de prensa con los comisarios de exposiciones artísticas. En todos los casos, la parte hispanohablante estaba representada por los medios de comunicación nacionales y extranjeros acreditados. Para la ISSPP, nos basamos en la tarea diaria llevada a cabo por la intérprete de un centro hospitalario de una zona turística, con alemán, danés, español, finlandés (lengua materna), inglés, noruego y sueco, como lenguas de trabajo y que interpreta entre el personal médico y los pacientes extranjeros constituidos en su mayoría por jubilados y turistas del norte de Europa. En ambos casos, los intérpretes entrevistados tienen experiencia superior a veinticinco años de ejercicio profesional.

\subsection{Metodología}

La segunda etapa de la metodología ha consistido en cumplimentar con los intérpretes la plantilla mencionada para análisis de situaciones mediadas, en el marco de entrevistas semiestructuradas en las que los intérpretes, guiados por esta herramienta metodológica, proporcionaron su autopercepción de las situaciones en las que intervinieron desde un punto de vista cualitativo.

Semi-structured interviews feature a core of common questions around which interaction in response to the interviewee's framing of central interview issues is allowed to emerge. The focus here is on the elicitation of perceptions, beliefs, or motives (Böser 2016: 236). 
Aunque no se trate de un cuestionario propiamente dicho, sino de orientar a los sujetos cumplimentando unas fichas, el método seguido permite obtener el feed-back retrospectivo y cualitativo de los intérpretes, acerca de los factores de los eventos comunicativos del corpus en los que han intervenido $y$, sobre todo, detectar aquellos potencialmente percibidos como inductores de estrés. Este procedimiento ha permitido mantener la atención en el tema central del estrés, desarrollando las respuestas guiadas al cumplimentar las fichas de análisis. "The unstructured interview maintains the focus on the central research question but will develop conversationally, allowing for interviewee-led contributions" (Böser 2016: 236). Así, se ha guiado a los sujetos en el exhaustivo trabajo de la cumplimentación de las tres fichas, en las que se incorporó la información proporcionada por los intérpretes en función de su experiencia y en respuesta a preguntas como: ¿qué medios técnicos utilizó? ¿Qué técnica de interpretación empleó? (Tabla 1, ítems 3 y 4, respectivamente), ¿qué interlocutores interactuaban en la situación comunicativa? (Tabla 2, ítem 1), ¿qué intercambios o contactos se mantuvieron antes y después del evento? (Tabla 3, ítems 3 y 4 respectivamente), o ¿qué textos u obras se mencionaron durante el encuentro? (Tabla 3, ítem 6). En este trabajo, por las lógicas limitaciones de espacio, se presentan las fichas en cuestión (Tablas 1 , 2 y 3), con las respuestas de los intérpretes resumidas. El caso de ISSPP en medio hospitalario está recogido por Miranda Rodríguez (2015: 26-38). Esta metodología ha permitido, en primer lugar, tipificar y caracterizar detalladamente los encuentros y, en segundo, identificar los factores que actúan como inductores de estrés en uno u otro tipo de interpretación, IC o ISSPP.

\subsection{Resultados}

Como última etapa del procedimiento metodológico, se han estudiado las respuestas de los intérpretes y sintetizado en tablas comparativas entre los dos tipos de interpretación, con la intención de presentar resultados sucintos y legibles. Estas tablas reflejan los contenidos de la herramienta empleada para el análisis, con sus ítems numerados. La primera columna de las tablas recoge los aspectos de la situación mediada contemplados; la segunda, las respuestas de cómo inciden en la IC; y la tercera, en la ISSPP. Cada tabla aparece seguida por un breve comentario de los resultados. 
3.3.1. Tabla 1. Factores situacionales

\begin{tabular}{|l|l|l|}
\hline \multicolumn{1}{|c|}{ Factores situacionales } & \multicolumn{1}{|c|}{ IC } & \multicolumn{1}{|c|}{ ISSPP } \\
\hline 1. Unidad espacial & $\begin{array}{l}\text { Compartida: presencia física } \\
\text { del intérprete }\end{array}$ & $\begin{array}{l}\text { Compartida: presencia física } \\
\text { del intérprete }\end{array}$ \\
\hline 2. Unidad temporal & $\begin{array}{l}\text { Una sesión de rueda de } \\
\text { prensa }\end{array}$ & $\begin{array}{l}\text { Una jornada de trabajo } \\
\text { (ronda de planta del } \\
\text { médico) }\end{array}$ \\
\hline $\begin{array}{l}\text { 3. Medios técnicos emple- } \\
\text { ados }\end{array}$ & $\begin{array}{l}\text { Micrófonos } \\
\text { Medios para grabación } \\
\text { audiovisual }\end{array}$ & $\begin{array}{l}\text { No disponibles, pero } \\
\text { serían necesarios por ruido } \\
\text { ambiente }\end{array}$ \\
\hline 4. Técnica de interpretación & $\begin{array}{l}\text { Interpretación bilateral (solo } \\
\text { 2 lenguas) } \\
\text { Posible interpretación } \\
\text { consecutiva por razones de } \\
\text { formalidad. Interpretación } \\
\text { susurrada de las preguntas } \\
\text { a la persona entrevistada }\end{array}$ & $\begin{array}{l}\text { Interpretación bilateral } \\
\text { (numerosas lenguas) } \\
\text { Posible IS de preguntas del } \\
\text { médico por exigencias de } \\
\text { rapidez }\end{array}$ \\
\hline
\end{tabular}

A primera vista se puede observar que ambas situaciones de interpretación comparten el aspecto de que el intérprete está presente físicamente y comparte el espacio con sus interlocutores. En ISSPP hay mayor volumen horario de trabajo; se realiza en peores condiciones acústicas siguiendo al médico en su ronda cotidiana por cada planta del hospital; y el manejo continuo de diferentes lenguas extranjeras en función de los pacientes, parecen factores susceptibles de generar, según la intérprete consultada, un mayor estrés. Estas respuestas de la intérprete entrevistada parecen corroborar la falta de definición de las condiciones laborales en este ámbito. No obstante, el estrés en IC puede venir inducido por la presencia de medios audiovisuales que graban y retransmiten la rueda de prensa para el público en general. Este aspecto dista mucho de ser desdeñable, puesto que multiplica la exposición de la tarea del intérprete cuya repercusión mediática le posibilitaría evaluar la calidad de su prestación. Alonso Araguás, Zapatero Santos (2019: 261-265) indican numerosos casos de errores de interpretación de los que se hacen eco los medios. Además, cabe destacar que en las ruedas de prensa contempladas se recurre a la interpretación consecutiva monológica (Jiménez Ivars 2002: 3), por razones, entre otras posibles, de grado elevado de formalidad o protocolo, y se hace interpretación susurrada de las preguntas de la prensa al entrevistado. En efecto, en cuanto a las ruedas de prensa: 
The full range of interpreting modes may be found in football press conferences, but the most common choice is consecutive interpreting (sometimes in combination with whispered interpreting), because it is relatively easy to organise and does not require any specialised equipment (Sandrelli 2015: 92).

Sin embargo, en las situaciones de ISSPP la intérprete, dada su experiencia, opta por la IS con el fin de contribuir a la rápida cadencia en las visitas médicas de la ronda cotidiana a los pacientes.

\subsubsection{Tabla 2. Factores interpersonales}

\begin{tabular}{|l|l|l|}
\hline Factores interpersonales & IC & ISSPP \\
\hline $\begin{array}{l}\text { 1. Identificación de los inter- } \\
\text { locutores }\end{array}$ & $\begin{array}{l}\text { Actores de prestigio } \\
\text { internacional o comisarios } \\
\text { de exposición y medios de } \\
\text { comunicación }\end{array}$ & $\begin{array}{l}\text { Médicos y pacientes } \\
\text { hospitalizados de edad } \\
\text { avanzada }\end{array}$ \\
\hline $\begin{array}{l}\text { 2. Bagaje cognitivo de los } \\
\text { interlocutores sobre el tema }\end{array}$ & $\begin{array}{l}\text { Desequilibrio de } \\
\text { conocimientos temáticos: } \\
\text { el compareciente ante } \\
\text { la prensa conoce mejor } \\
\text { su actividad profesional } \\
\text { o personal }\end{array}$ & $\begin{array}{l}\text { Desequilibrio de } \\
\text { conocimientos temáticos: } \\
\text { el profesional médico tiene } \\
\text { mayor bagaje cognitivo } \\
\text { y pregunta a personas que } \\
\text { no conocen el tema }\end{array}$ \\
\hline 3. Objetivos comunicativos & $\begin{array}{l}\text { Coincidentes: ambas partes } \\
\text { quieren dar a conocer } \\
\text { la actividad profesional } \\
\text { o personal del entrevistado }\end{array}$ & $\begin{array}{l}\text { Coincidentes: ambas partes } \\
\text { desean resolver el problema } \\
\text { de salud en cuestión }\end{array}$ \\
\hline 4. Estatus de poder social & Equilibrado & Desequilibrado \\
\hline 5. Homogeneidad cultural & Equilibrada: origen europeo & $\begin{array}{l}\text { Equilibrada: orígenes } \\
\text { europeos diversos }\end{array}$ \\
\hline
\end{tabular}

La plantilla que recoge los factores personales y socioculturales relativos a los interactantes, así como sus motivos y objetivos, muestra claramente que, aunque el estrés pueda estar presente en ambos contextos, la propia naturaleza de la situación comunicativa médica, de los interlocutores implicados y su estado de enfermedad, exigen del intérprete un mayor esfuerzo añadido de adaptación y explicitación continua de los contenidos médicos para compensar el desequilibrio de conocimientos temáticos. En este caso de ISSPP, la transmisión eficaz de la información resulta esencial de cara al estado de los pacientes, mientras que no ocurre lo mismo en la situación de IC, en la que el desequilibrio de nivel de conocimientos entre los participantes no conlleva consecuencias personales, sino profesionales. Ocasionalmente, los medios de comunicación, podrían seguir preguntando o completando posteriormente 
su búsqueda documental, si fuera necesario. Por consiguiente, parece quedar claro que la propia índole de la situación comunicativa y los interlocutores implicados en ella son susceptibles de transferir sentimientos y emociones (Blair, Ramones 1996: 24) al intérprete que se estresaría a causa de un componente fundamentalmente emocional en ISSPP y no así en IC.

Estudios reseñados y la propia experiencia indican que los traductores e intérpretes en los servicios públicos deben enfrentarse con frecuencia a situaciones que pueden provocarles un fuerte impacto emocional y psicológico, el cual puede derivar en graves consecuencias (Valero Garcés 2006: 153).

Además, con respecto a la diferencia de estatus de poder entre los interlocutores, la persona entrevistada beneficiaría del protagonismo, incluso de un cierto narcisismo, en el caso de actores que nos ocupa, o de un tanto de cautela, si se tratase de políticos que compareciesen ante la prensa que, por su parte, ostenta el poder de crear opinión y encumbrar o derrumbar ídolos. No obstante los matices anteriores, en las situaciones contempladas de ISSPP, la diferencia de nivel de conocimientos especializados entre el profesional médico y el beneficiario de la asistencia sanitaria en situación de enfermedad y, por tanto, dependiente de la atención del primero, permitiría imbuir al médico de una cierta autoridad, lo que parece corroborar el desequilibrio de estatus entre interlocutores característico de la ISSPP.

Por último, acerca de la coincidencia de culturas occidentales entre interlocutores, en otras situaciones de ISSPP, por ejemplo, en el campo de extranjería o policial, este parámetro también debe constituir, sin duda, un factor de estrés por su disparidad y una característica esencial de otros contextos de ISSPP (El-Madkouri Maataoui 2008, Taibi, El-Madkouri Maataoui 2016).

\subsubsection{Tabla 3. Factores informacionales}

\begin{tabular}{|l|l|l|}
\hline \multicolumn{1}{|c|}{ Factores informacionales } & \multicolumn{1}{|c|}{ IC } & \multicolumn{1}{c|}{ ISSPP } \\
\hline $\begin{array}{l}\text { 1. Tema y grado de } \\
\text { especialización }\end{array}$ & $\begin{array}{l}\text { Cine y arte } \\
\text { Alto grado de especializa- } \\
\text { ción }\end{array}$ & $\begin{array}{l}\text { Medicina y salud } \\
\text { Alto grado de } \\
\text { especialización }\end{array}$ \\
\hline $\begin{array}{l}\text { 2. Cantidad o densidad de } \\
\text { información }\end{array}$ & Abundante y previsible & $\begin{array}{l}\text { Abundante e imprevisible } \\
\text { (incluso tema por dolencia } \\
\text { y lengua de cada paciente) }\end{array}$ \\
\hline $\begin{array}{l}\text { 3. Intercambios de } \\
\text { información previos al } \\
\text { encuentro }\end{array}$ & $\begin{array}{l}\text { Contactos con organización } \\
\text { para preparación del tema }\end{array}$ & $\begin{array}{l}\text { Sin preparación por no ser } \\
\text { personal médico y rotación } \\
\text { continua de pacientes }\end{array}$ \\
\hline
\end{tabular}


3.3.3. Tabla 3. continuación

\begin{tabular}{|l|l|l|}
\hline \multicolumn{1}{|c|}{ Factores informacionales } & \multicolumn{1}{|c|}{ IC } & \multicolumn{1}{c|}{ ISSPP } \\
\hline $\begin{array}{l}\text { 4. Intercambios de } \\
\text { información posteriores al } \\
\text { encuentro }\end{array}$ & $\begin{array}{l}\text { Publicación en medios que } \\
\text { se hacen eco de la prestación } \\
\text { del intérprete }\end{array}$ & $\begin{array}{l}\text { Realización de otras } \\
\text { funciones: trámites médicos } \\
\text { y administrativos con } \\
\text { compañías de seguros }\end{array}$ \\
\hline $\begin{array}{l}\text { 5. Comportamiento no } \\
\text { verbal }\end{array}$ & $\begin{array}{l}\text { Equilibrado entre profesio- } \\
\text { nales }\end{array}$ & $\begin{array}{l}\text { Equilibrado } \\
\text { Mímica esencial para } \\
\text { comprensión por parte de } \\
\text { los enfermos }\end{array}$ \\
\hline $\begin{array}{l}\text { 6. Textos o realizaciones } \\
\text { citados en el encuentro }\end{array}$ & $\begin{array}{l}\text { Películas, festivales de cine, } \\
\text { obras de arte... }\end{array}$ & $\begin{array}{l}\text { Diagnósticos y tratamientos } \\
\text { médicos, consentimientos } \\
\text { informados para pruebas } \\
\text { médicas, formularios } \\
\text { administrativos... } \\
\text { (Traducción a la vista) }\end{array}$ \\
\hline $\begin{array}{l}\text { 7. Textos o realizaciones } \\
\text { secundarios para el } \\
\text { encuentro }\end{array}$ & $\begin{array}{l}\text { Noticias en medios de } \\
\text { comunicación }\end{array}$ & $\begin{array}{l}\text { Gestiones administrativas } \\
\text { con hospital y aseguradoras }\end{array}$ \\
\hline
\end{tabular}

En cuanto al contenido informativo del encuentro, parece obvio que cualquier tema puede resultar altamente especializado. Sin embargo, dados la situación comunicativa y el perfil de los interlocutores en cuestión, el tema y su grado de especialización en la ISSPP es susceptible de generar mayor estrés, sobre todo, porque son problemáticas de gran implicación personal, cuya resolución es vital para una de las partes, y porque, con frecuencia, se deben afrontar en condiciones de improvisación sin preparación previa, puesto que la intérprete desconoce la dolencia e, incluso, la lengua de los pacientes de nuevo ingreso en cada ronda diaria.

Asimismo, se añaden dos aspectos fundamentales de cara a nuestro análisis que ilustran otras funciones que se deben realizar en ISSPP. La disparidad de conocimientos entre interactantes exige que la intérprete explique los contenidos a los interlocutores con menos conocimientos, los pacientes, sin descartar el recurso a la mímica, imágenes o dibujos, lo que puede representar un factor de estrés, dada su relevancia para asegurar la comunicación.

En segundo lugar, el tratamiento de los textos con los que se trabaja en uno u otro tipo de interpretación, ya que en la ISSPP se requieren nuevas prestaciones al intérprete, a saber, la traducción a la vista y escrita de textos un tanto estandarizados, como consentimientos informados o formularios administrativos; trámites administrativos con aseguradoras en diferentes idiomas; e, incluso, llamadas a familiares de los pacientes. Todo ello no puede 
más que suponer un nuevo factor de estrés al exigir otras funciones al profesional, por si fuera poco, con textos especializados (médicos y administrativos), cuya mala traducción podría acarrear graves consecuencias para la vida de los interlocutores (problemas de salud y económicos).

Este último aspecto parece derivar de la menor consolidación de este perfil de intérprete y de su estatus como empleado del centro hospitalario, frente al profesional autónomo de conferencias, perfil que cuenta ya con casi un siglo de tradición en la era contemporánea, pero cuyos representantes tuvieron que luchar también por conquistar el reconocimiento de sus condiciones laborales (Baigorri Jalón 2014).

\section{Conclusiones}

Con el estudio de los factores constituyentes de los casos de IC e ISSPP, que hemos pretendido mostrar aquí mediante una sucinta esquematización en tablas, hemos identificado los factores que, en mayor medida, pueden ser fuente de estrés en uno u otro tipo de interpretación.

Si en ambos casos, el intérprete está expuesto ante los interlocutores al compartir su espacio, en la situación de IC la grabación de los medios audiovisuales le expone ante un público más amplio, lo que puede representar un motivo de estrés. En el caso estudiado de ISSPP en el ámbito hospitalario, parecen ser las condiciones horarias y acústicas las que más estresan, según los comentarios obtenidos.

En términos generales, los perfiles socioculturales, así como los motivos y objetivos comunicativos de los interlocutores, son coincidentes, en IC y en ISSPP, a diferencia de otros contextos de ISSPP, por ejemplo, de extranjería o policiales. No obstante, la propia situación comunicativa o contexto socioprofesional, las características de los interlocutores (médicos y pacientes) y la naturaleza del tema hacen que se dé una mayor asimetría de conocimientos y social en el caso de ISSPP. Estos aspectos son susceptibles de provocar estrés emocional en el intérprete por empatía con el interlocutor más indefenso.

En cuanto a los aspectos informacionales, si bien en ambas situaciones los temas pueden resultar altamente especializados (cine, arte, medicina, seguros), en estos casos de IC se produjeron contactos previos para organizar las ruedas de prensa, mientras que en el contexto de la ISSPP estudiada los profesionales médicos no parecen integrar a la intérprete en su rutina diaria y esta debe afrontar las eventuales dificultades temáticas y lingüísticas con 
un mayor grado de improvisación, cuando resulta obvio que la preparación y documentación previas son una de las mejores estrategias de los intérpretes.

Asimismo, además de los distintos factores mencionados arriba como potenciales estresores en uno u otro contexto, cabe destacar que en el caso concreto estudiado de ISSPP se requiere a la intérprete otras tareas, como la traducción a la vista de documentos durante el encuentro mediado y, después del evento, la gestión de trámites hospitalarios, administrativos con compañías de seguros e, incluso, sociales con los familiares de los pacientes. En este contexto de ISSPP hospitalaria, la ejecución de estas funciones viene a añadir, pues, otras competencias a las que se ponen en juego durante la ejecución del proceso interpretativo (PACTE 2003, 2014), habituales en IC para una prestación de interpretación consecutiva dialógica. Esas otras destrezas solicitadas a la intérprete y observadas en el análisis de nuestro estudio, son las que intentamos reflejar a continuación:

- Capacidad de mediación social. Debido a uno de los factores más desequilibrados, la diferencia de conocimientos temáticos entre médicos y pacientes (Tabla 2, ítem 2), el profesional médico adquiere un estatus superior (Tabla 2, ítem 4) frente al desamparo de jubilados enfermos en un país extranjero del que no conocen la lengua ni la cultura. Así, la intérprete consultada menciona la mediación con las familias de lengua extranjera de las personas hospitalizadas y su adaptación constante al método de trabajo de cada médico (especialistas o generalistas, dependiendo de la patología del paciente) y a la situación personal de cada paciente (alterada por su enfermedad) (Tabla 3, ítems 2 y 3), propiciando la confianza y la comprensión mutuas, para lo que se recurre incluso a gestos o mímica (Tabla 3 , ítem 5).

- Capacidad para gestionar el intercambio informativo. Las preguntas del profesional médico se orientan a recabar el máximo posible de información pertinente (Tabla 3, ítem 2) en el menor tiempo disponible durante su ronda de planta cotidiana (Tabla 1, ítem 2). Por lo tanto, la intérprete entrevistada manifiesta tener que redirigir al paciente que comienza a dar más información de la necesaria, como quejas o dudas, con lo que gestiona también los turnos de palabra.

- Gestión del propio estrés emocional. La intérprete empatiza con los pacientes, compadeciéndolos (Tabla 2, ítem 1: personas mayores hospitalizadas), lo que le hace tender a sobrepasar sus funciones de mediación lingüística para intentar consolarlos y ayudarlos.

- Empleo activo de varias lenguas de trabajo (Tabla 3, ítem 2), al cambiar de un paciente a otro (Tabla 3 , ítem 3 ), y conocimientos especializados y léxicos de medicina. 
- Traducción a la vista (Tabla 3, ítem 6) de documentos médicos especializados y administrativos con menor grado de especialización.

- Gestión administrativa de trámites hospitalarios y con las compañías aseguradoras (Tabla 3, ítem 7).

Por consiguiente, en los casos contemplados tanto de IC como de ISSPP, los intérpretes comparten una serie de competencias comunes referentes a la prestación de interpretación consecutiva dialógica en sí. A pesar de ello, exceptuando el requisito de conocimientos lingüísticos en varios idiomas y temáticos especializados o el recurso a la traducción a la vista, que pueden darse en contextos de IC, los demás aspectos reseñados arriba exceden claramente las competencias habituales en IC y, de ese modo, en ISSPP se requieren también al intérprete otras aptitudes que distan de las del perfil profesional de intérprete de IC.

En conclusión, se podría afirmar que la intérprete, en este contexto de ISSPP, contribuye a asegurar la prestación del servicio hospitalario mediante su participación activa, lo que pondría en entredicho la supuesta neutralidad del intérprete, puesto que gestiona relaciones humanas y administrativas, así como el propio intercambio comunicativo.

Así, aunque conscientes de los límites del estudio de casos llevado a cabo que incluye una muestra reducida de situaciones de interpretación, se podría concluir con respecto a la hipótesis inicial que, al tratarse de situaciones de interpretación consecutiva dialógica, existen muchos factores similares entre ambos contextos susceptibles de inducir estrés, tal y como se recopila en las tablas anteriores. No obstante, la intérprete de ISSPP se ve obligada a ejecutar otras funciones que pueden generar no más ni menos estrés que en IC, sino un estrés que comporta otros aspectos, mayormente psico-afectivos y achacables a las condiciones de trabajo, puesto que los vínculos entre los factores cognitivo-afectivos y las competencias en interpretación son sumamente estrechos (Brisau, Godjins, Meuleman 1994: 87).

\section{Bibliografía}

Abril Martí I., 2004, La interpretación social. Una profesión por derecho propio, [in:] Acerca de la traducción y la interpretación, V. Pacheco Costa (ed.), Sevilla, pp. 213-228.

Abril Martí I., 2006, La Interpretación en los Servicios Públicos. Caracterización como género, contextualización y modelos de formación. Hacia unas bases para el diseño curricular, Granada, https://digibug.ugr.es/ 
bitstream/handle/10481/1075/16235320.pdf?sequence=1\&isAllowed=y (entry: 8.02.2019).

Achotegui J., 2009, Estrés límite y salud mental. El síndrome del inmigrante con estrés crónico y múltiple (síndrome de Ulises), "Gaceta Médica de Bilbao", 106, pp. 122-133.

AIIC (International Association of Conference Interpreters), 2002, Interpreter workload study. Full report, http://aiic.net/p/657 (entry: 12.02.2019).

Alexieva B., 1997, A typology of interpreter-mediated events, "The Translator", 3(2), pp. 153-174.

Alonso Araguás I., Zapatero Santos P., 2019, La interpretación en competiciones de fútbol internacionales. Un estudio de caso: la UEFA, "SENDEBAR", 30, pp. 245-271.

Baigorri Jalón J., 2014, From Paris to Nuremberg. The birth of conference interpreting, Amsterdam-Philadelphia.

Bendazzoli C., 2010, Testi e contesti dell'interpretazione di conferenza. Uno studio etnografico, Bolonia.

Blair T., Ramones V., 1996, Understanding vicarious traumatization, "Journal of Psychosocial Nursing", 34, pp. 24-30.

Bobaila I., 2011, Interpretación vs. mediación cultural - referencias al ámbito educativo español, "Studia Universitatis Babes Bolyai Philologia", 56(1), pp. 191-197.

Böser U., 2016, Interviews and focus groups, [in:] Researching translation and intepreting, C. V. Angelelli, B. J. Baer (eds.), London-New York, pp. 236-246.

Brisau A., Godjins R., Meuleman C., 1994, Towards a psycholinguistic profile of the interpreter, "Meta", 39(1), pp. 87-94.

Collados Aís A., Fernández Sánchez M. M., Stévaux E., 2001, Concepto, técnicas y modalidades de interpretación, [in:] Manual de interpretación bilateral, A. Collados Aís, M. M. Fernández Sánchez (eds.), Granada, pp. 39-60.

Corsellis A., 2010, Traducción e interpretación en los servicios públicos. Primeros pasos, Granada.

Darias Marrero A., 2006, Interpretación. Tipos de situación comunicativa y didáctica, tesis de doctorado sin publicar, Universidad de Las Palmas de Gran Canaria.

Darias Marrero A., 2009, La situación comunicativa y sus constituyentes en interpretación, [in:] Estudios de traducción. Perspectivas, S. Bravo Utrera, R. García López (eds.), Frankfurt am Main, pp. 32-49.

Darias Marrero A., Vidal Luengo A. R., 2018, Formación asistida por orde- 
nador para la interpretación y la mediación intercultural en Servicios Públicos (árabe-español), [in:] Casos de éxito en aprendizaje ubicuo y social mediado con tecnologías, A. I. Allueva Pinilla, J. L. Alejandre Marco (eds.), Zaragoza, pp. 193-201.

El-Madkouri Maataoui M., 2008, Lengua oral y lengua escrita en la traducción e interpretación en los servicios públicos, "Tonos Digital”, 15, http://www.tonosdigital.es/ojs/index.php/tonos/article/viewArticle/183 (entry: 20.03.2018).

Gile D., 1989, Les flux d'information dans les réunions interlinguistiques et l'interprétation de conférence. Premières observations, "Meta", 34(4), pp. 649-660.

Ilg D., Lambert S., 1996, Teaching consecutive interpreting, "Interpreting", $1(1)$, pp. 69-99.

Jiménez Ivars M.A., 2002, Variedades de interpretación: modalidades y tipos, "Hermēneus. Revista de Traducción e Interpretación", 4, pp. 95-114.

Jiménez Ivars M. A., 2012, Primeros pasos hacia la interpretación InglésEspañol, Castelló de la Plana.

Jiménez Ivars M. A., Pinazo Calatayud D., 2002, Aptitudes necesarias en la formación de intérpretes. Un estudio exploratorio, "Quaderns. Revista de traducció", 8, pp. 77-97.

Kurz I., 2003, Physiological stress during simultaneous interpreting. A comparison of experts and novices, "The Interpreters' Newsletter", 12, pp. 51-67.

Lazarus R. S., 2000, Estrés y emoción. Manejo e implicaciones en nuestra salud, Bilbao.

Lvóvskaya Z., 1997, Problemas actuales de la traducción, Granada.

Miranda Rodríguez M., 2015, El estrés en la interpretación: análisis de un caso de interpretación para los servicios públicos, trabajo de fin de Grado sin publicar, Universidad de Las Palmas de Gran Canaria.

Moser-Mercer B., Künzli A., Korac M., 1998, Prolonged turns in interpreting. Effects on quality, physiological and psychological stress (Pilot study), "Interpreting", 3(1), pp. 47-64.

PACTE, 2003, Building a translation competence model, [in:] Triangulating translation. Perspectives in process oriented research, F. Alves (ed.), Amsterdam, pp. 43-66.

PACTE, 2014, First results of PACTE group's experimental research on translation competence acquisition: The acquisition of declarative knowledge of translation, "MonTI. Monografías de Traducción e Interpretación", vol. especial 1, pp. 85-115. 
Pöchhacker F., 2003, Enfoque funcional de la interpretación simultánea, [in:] Avances en la investigación sobre interpretación, A. Collados Aís, J. A. Sabio Pinilla (eds.), Granada, pp. 105-122.

Pöchhacker F., 2004, Introducing interpreting studies, London-New York.

Pöllabauer S., 2012, Gatekeeping practices in interpreted social service encounters, "Meta", 571, pp. 213-234.

Renau Michavila M., 2010, La técnica Alexander, una nueva voz en la didáctica de interpretación, "Puentes", 9, pp. 81-88.

Riccardi A., Marinuzzi G., Zecchin S., 1998, Interpretation and stress, “The Interpreters' Newsletter", 8, pp. 93-106.

Roberts R., 1994, Community interpreting today and tomorrow, [in:] Proceedings of the $35^{\text {th }}$ Annual Conference of the American Translators Association, P. Krawutschke (ed.), Medford, pp. 127-138.

Sandrelli A., 2015, And maybe you can translate also what I say. Interpreters in football press conferences, “The Interpreters' Newsletter”, 20, pp. 87-105.

Setton R., Dawrant A., 2016, Conference interpreting. A complete course, Amsterdam-Philadelphia.

Taibi M., El-Madkouri Maataoui M., 2016, Interpreting taboo. The case of Arabic interpreters in Spanish Public Services, [in:] New insights into Arabic translation and interpreting, Taibi M. (ed.), Bristol-Buffalo-Toronto, pp. 69-86.

Ticca A. C., Traverso V., 2017, Participation in bilingual interactions. Translating, interpreting and mediating documents in a French social centre, "Journal of Pragmatics", 107, pp. 129-146.

Valero Garcés C., Dergam A., 2003, ¿Mediador social = mediador interlingüístico = Intérprete? Práctica, formación y reconocimiento social del intérprete en los Servicios Públicos, [in:] La evaluación de la calidad en interpretación. Docencia y profesión, A. Collados Aís, M. Fernández Sánchez, M. Pradas Macías, C. Sánchez Adam, E. Stévaux (eds.), Granada, pp. 257-266.

Valero Garcés C. (ed.), 2005, Traducción como mediación entre lenguas y culturas. Translation as mediation or how to bridge linguistic and cultural gaps, Alcalá de Henares.

Valero Garcés C., 2006, El impacto psicológico y emocional en los intérpretes y traductores de los servicios públicos. Un factor a tener en cuenta, "Quaderns. Revista de traducció", 13, pp. 141-154.

Valero Garcés C., 2008, Formas de mediación intercultural. Traducción e interpretación en los servicios públicos. Conceptos, datos, situaciones y práctica, Granada. 
Valero Garcés C., 2014, Health communication and multicultural communities. Topics on intercultural communication for healthcare professionals, Newcastle upon Tyne.

Vermeer H. J., 1983, Aufsätze zur Translationstheorie, Heidelberg.

Wadensjö C., 1992, Interpreting as interaction. On dialogue-interpreting in Immigration Hearings and Medical Encounters, Ph.D. diss., Linkoping. Wadensjö C., 1998, Interpreting as interaction, Londres-New York.

Witte H., 2000, Die Kulturkompetenz des Translators. Begriffliche Grundlegung und Didaktisierung, Tübingen.

Witte H., 2005, Traducir entre culturas. La competencia cultural como componente integrador del perfil experto del traductor, "SENDEBAR", 16, pp. 27-58.

\section{Stress in interpreter-mediated situations: a comparative study between conference interpreting and public service interpreting}

\section{Summary}

The present study analyzes the stress-inducing factors arising in two different interpreter-mediated situations where dialogue interpreters are involved. Specifically, the focus is on conference interpreting (press conferences) and public service interpreting (health interpreting). The methodology consists of three main steps. First, drawing on existing classifications of interpreter-mediated communicative situations, the main features characterizing the situations studied are singled out in line with situational, interpersonal, and informational factors. These factors make up the methodological tool applied in the two subsequent steps of the analysis, including semi-structured interviews and a thematic analysis of the answers obtained. The semi-structured interviews were conducted after the completion of the interpreting assignments under consideration. The interpreters were asked to complete a form indicating the stress-inducing factors and add comments. Finally, the answers obtained in the thematic analysis were collated and entered into tables, including a summary of the comments provided by the interpreters. The results concerning the conference interpreting situation are then discussed against the results from the public service interpreting situation. Overall, this leads to the conclusion that there are no major differences in the level of stress experienced in the two situations. However, there are discrepancies in the kinds of stress-inducing factors at stake in each situation. For instance, the conference interpreter feels stressed when they know their performance will face media exposure (e.g. through recording or streaming). On the other hand, the public service interpreter tends to empathize with the weaker party among their interlocutors, which results in emotional stress. Moreover, the public service interpreter is required to perform additional tasks (e.g. sight translation, written trans- 
lation of medical and administrative documents, administrative management with insurance companies) and becomes aware of the subject and the languages involved in each assignment at the very last moment. It is clear that the professional profile of conference interpreters is more established than that of public service interpreters, whereas public service interpreters perform multiple tasks that exceed linguistic and cultural mediation proper.

Keywords: interpreter-mediated situations, stress, conference interpreting, press conferences, public service interpreting, health interpreting 